\title{
BMJ Open Caesarean section in Palestine using the Robson Ten Group Classification System: a population-based birth cohort study
}

Mohammed Walid Zimmo, ${ }^{1,2,3}$ Katariina Laine, ${ }^{4,5}$ Sahar Hassan, ${ }^{6}$ Bettina Bottcher, ${ }^{7}$ Erik Fosse, ${ }^{2,3}$ Hadil Ali-Masri, ${ }^{2,3,8}$ Kaled Zimmo, ${ }^{2,3,9}$ Ragnhild Sørum Falk, ${ }^{10}$ Marit Lieng, ${ }^{2,11}$ Åse Vikanes ${ }^{3}$

To cite: Zimmo MW, Laine $\mathrm{K}$, Hassan $\mathrm{S}$, et al. Caesarean section in Palestine using the Robson Ten Group Classification System: a population-based birth cohort study. BMJ Open 2018;8:e022875. doi:10.1136/ bmjopen-2018-022875

- Prepublication history and additional material for this paper are available online. To view these files, please visit the journal online (http://dx.doi. org/10.1136/bmjopen-2017022875).

Received 10 March 2018 Revised 30 May 2018

Accepted 20 September 2018

D Check for updates

(C) Author(s) (or their employer(s)) 2018. Re-use permitted under CC BY-NC. No commercial re-use. See rights and permissions. Published by BMJ.

For numbered affiliations see end of article.

\section{Correspondence to} Dr Mohammed Walid Zimmo; mohammedzimm01983@gmail. com

\section{ABSTRACT}

Objective To analyse the current situation of caesarean section in Palestine using the Robson Ten Group

Classification System (TGCS).

Design A population-based birth cohort study.

Setting Obstetrical departments in three governmental hospitals in Gaza.

Participants All women (18 908) who gave birth between 1 January 2016 and 30 April 2017.

Methods The contributions of each group to the study population and to the overall rate of caesarean section were calculated, as well as the rate of caesarean section in each TGCS group. Differences in proportions between study hospitals were assessed by $\chi^{2}$ test.

Main outcome measures The main outcome was the contributions of each group to the overall caesarean section rate.

Results The overall rate of caesarean section was $22.9 \%$ (4337 of 18908 ), ranging from $20.6 \%$ in hospital 1 to $24.6 \%$ in hospital 3. The largest contributors to the overall caesarean section rate were multiparous women with single cephalic fullterm pregnancy who had undergone at least one caesarean section (group 5, 42.6\%), women with multiple pregnancies (group $8,11.6 \%$ ) and those with single cephalic preterm labour (group 10, 8.1\%). Statistically significant differences in caesarean section rates between the study hospitals were observed in group 1 (nulliparous women with single cephalic full-term pregnancy and spontaneous labour), group 4 (multiparous with single cephalic full-term pregnancy with induced labour or prelabour caesarean section), group 5 (multiparous with single cephalic full-term pregnancy with previous caesarean section) and in group 7 (multiparous with breech presentation).

Conclusion Women in groups 5, 8 and 10 were the largest contributors to the overall caesarean section rate in the study hospitals. Efforts to reduce the differences in obstetrical care between hospitals need to be directed towards increasing the proportion of vaginal births after caesarean section and by reducing primary caesarean section in multiple pregnancies and preterm labour.
Strengths and limitations of this study

- This study is the largest, population-based, prospective birth cohort study in Palestine.

- It was the first to explore caesarean section rates in Palestine using the Robson Ten Group Classification System.

- All women who gave birth in the study hospitals were included, reducing the risk for selection bias.

- The main limitation of this study was the fact that women, who gave birth in the West Bank or in the private sector in Gaza, were not included.

\section{INTRODUCTION}

Globally, the caesarean section rate is rising continuously, making caesarean section one of the most common surgical procedures. ${ }^{1}$ One in five pregnant women undergoes caesarean section. ${ }^{1}$ The caesarean section rate is often used as an indicator for the quality of healthcare and may therefore reflect improvement of clinical governance at national and international levels. However, caesarean section rates vary between countries and even between hospitals within the same country. ${ }^{1-3}$ The WHO recommends caesarean section rates to be between $10 \%$ and $15 \% .^{4}$ In order to investigate the underlying mechanisms for the global rise in caesarean section rates, it is fundamental to identify which groups of women are at higher risk to undergo caesarean section. For this reason, a classification system that can monitor and compare caesarean section rates in a standardised, reliable and consistent manner has been established. ${ }^{5}$ The International Federation of Gynecology and Obstetrics and WHO recommend the Robson Ten Group Classification System (TGCS) as a global standard for assessing, monitoring and comparing caesarean 
section rates between countries and institutions. ${ }^{6-8}$ The TGCS classifies women into 10 groups according to five obstetrical characteristics that are routinely documented and easy to implement (table 1 ). ${ }^{5}$ By applying TGCS, caesarean section births are being registered in relation to the women's and pregnancies' characteristics rather than medical indications. ${ }^{56}$

In Palestine, and particularly in Gaza, pregnant women receive regular antenatal care by antenatal clinics run by the United Nations Relief and Works Agency, the Palestinian Ministry of Health or private clinics. Care for women giving birth is offered in governmental and private hospitals. Governmental health services are available in all geographical areas and offer services with governmental insurance cover at very low cost. ${ }^{9}$ Hence, the majority $(73.0 \%)$ of births in Gaza take place in the governmental hospitals. ${ }^{910}$ The caesarean section rates in the governmental hospitals ranged from $16.6 \%$ to $26.0 \%$ in $2015 .^{9}$ The fertility rate, although falling, is currently still high in Gaza with 4.5, leading to around 55000 births every year. ${ }^{910}$ This leads to a large workload on labour and delivery wards in the Gaza-Strip which are generally poorly equipped and do not offer single rooms, except for specific cases. Furthermore, staff numbers are low and stretched by the current workload. ${ }^{10}$ Therefore, one-to-one care, which is an important intervention to achieve pain management as well as to prevent caesarean sections, is not available on the labour wards of governmental hospitals in Gaza. ${ }^{10}$

In Palestine, no hospital has used the TGCS so far. The objective of this study was to analyse the current situation of caesarean sections with use of the TGCS, and thus to identify the main contributors to the caesarean section rates in three hospitals in Gaza.

\section{Table 1 The Robson Ten Group Classification System}

\section{Group Description}

1 Nulliparous, singleton, cephalic, full-term, spontaneous labour.

2 Nulliparous, singleton, cephalic, full-term, induced labour or prelabour caesarean section.

3 Multiparous, singleton, cephalic, full-term, without a previous caesarean section, spontaneous labour.

4 Multiparous, singleton, cephalic, full-term, without a previous uterine scar, induced labour or prelabour caesarean section.

\begin{tabular}{ll}
\hline 5 & $\begin{array}{l}\text { Multiparous, singleton, cephalic, full-term, with a } \\
\text { previous caesarean section. }\end{array}$ \\
\hline 6 & Nulliparous, singleton, breech. \\
7 & Multiparous, singleton, breech. \\
8 & Multiple pregnancy (twins or higher-order multiples). \\
9 & Singleton, transverse or oblique lie. \\
10 & Singleton, cephalic, preterm. \\
\hline
\end{tabular}

\section{METHODS}

\section{Study design and participants}

The data were obtained from a population-based birth cohort study in three Palestinian governmental hospitals in Gaza from 1 January 2016 to 30 April 2017. Two of the hospitals were teaching hospitals (hospitals 2 and 3). Teaching hospitals in Palestine have educational programmes for health personnel; such as medical doctors, midwives and nurses. Two of the hospitals were referral hospitals (hospitals 1 and 3). Referral hospitals in Palestine receive patients from other private or governmental hospitals in the neighbouring areas. Hospital 2, being non-referral, was the only one without a maternal intensive care unit. Further characteristics of the study hospitals are presented by Sahar et al. ${ }^{11}$

All women, who gave birth in the study hospitals during the study period were eligible for inclusion. Cases with unknown mode of delivery $(n=373)$ or cases with missing information on one or more of the following variables were excluded: parity, presentation, gestational age or previous caesarean section ( $n=3$ ) (figure 1).

A case registration form, developed by Palestinian and Norwegian obstetricians and midwives, was used to collect data on mode of delivery, parity, presentation, gestational age and history of previous caesarean section. ${ }^{11}$ Before the data collection started, research teams in each study hospital were established, comprising the heads of obstetrical departments, medical doctors and midwives working in the labour wards. The case registration form was filled in by medical doctors or midwives who attended the births. The registered data were entered by research teams into a tailor-made version of the District Health Information Software 2 (V.2.24) which had been created by the Department of Global Infrastructure at the University of Oslo. Then data were transferred to be stored in Service for Sensitive Data platform which is developed and operated by the University of Oslo for researchers to collect, store, analyse and share sensitive data in compliance with the Norwegian regulations regarding individuals' privacy.

\section{Patients and public involvement}

There was no patient or public involvement in planning or executing this study. There are no plans to disperse the results of our research to study participants or the applicable patient community. However, results are being disseminated among the professional communities of Palestine and to policy-makers, with the intent to inform future health policy decisions.

\section{The Robson Ten Group Classification System}

All women were classified according to the TGCS based on the following characteristics: (1) parity (nulliparity/ multiparity/multiparity with previous caesarean section), (2) number of fetuses (single/multiple), (3) presentation of the fetus (cephalic/breech/transverse), (4) onset of labour (spontaneous/induced/prelabour caesarean section), (5) gestational age (term or preterm) (table 1). 


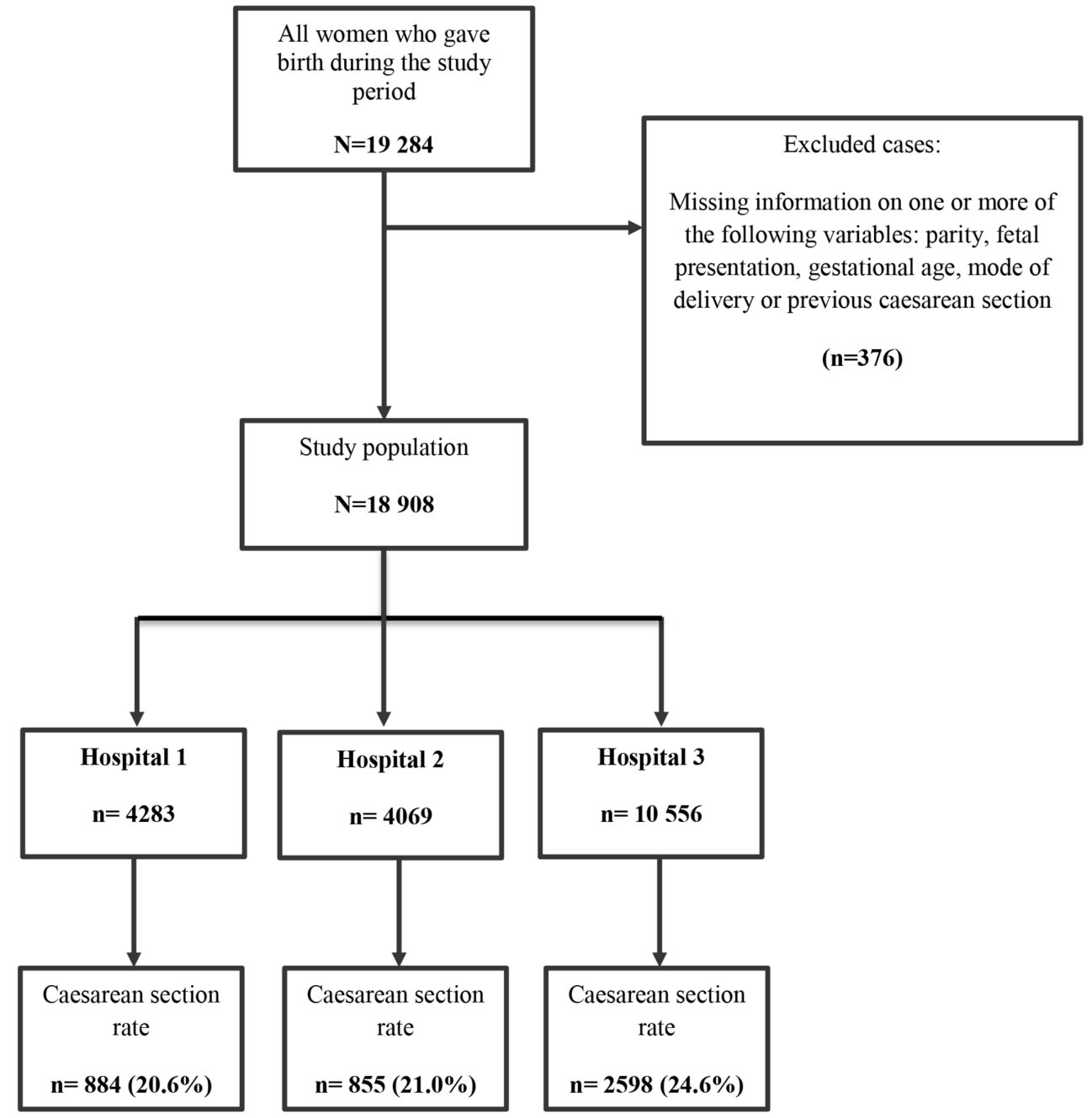

Figure 1 Flow chart of the study population, multicentre study from Palestine (from 1 January 2016 to 30 April 2017).

Nulliparity was defined as the woman giving birth for the first time, and multiparity as the woman having had one previous birth or more. Term pregnancy was defined as having completed 37 gestational weeks or more, whereas preterm pregnancy was defined as less than 37 completed gestational weeks. Induction of labour was defined as the use of any medication, amniotomy or cervical balloon, when women were not in labour. Caesarean section rates were calculated as number of caesarean sections divided by the number of births in the study population. This was calculated for the total population to find the overall caesarean section rate as well as separately for each study hospital and TGCS group.

\section{Outcome}

The primary outcome was the contributions of each group to the overall caesarean section rate. The secondary outcome was to identify the main contributors to the caesarean section rates in three hospitals in Gaza, and explore differences between hospitals in the contributions of each group to the overall caesarean section rate.

\section{Statistics}

Descriptive statistics were presented as frequencies and proportions. Number of births and proportion of caesarean section within each group of the TGCS were presented, and further stratified by the three study hospitals.

To assess differences in proportions of caesarean section by hospitals, $\chi^{2}$ tests within each TGCS group were performed. $\mathrm{P}$ values $<0.05$ were considered statistically significant. All statistical analyses were performed using SPSS V.24.

\section{RESULTS}

From the total number of births (18 908), 22.6\% took place in hospital $1,21.5 \%$ in hospital $2 \%$ and $55.8 \%$ in hospital 3. The majority of women were aged between 21 and 30 years. Hospital 2 had the largest proportion $(54.8 \%)$ of women with maternal age $\leq 20$ years. Almost $70 \%$ of women were multiparous (online supplementary table 1). 
Table 2 Number of births in each group of the Robson Ten Group Classification System in the study hospitals ( $n=18908)$

\begin{tabular}{lcccc}
$\begin{array}{l}\text { Robson Ten Group } \\
\text { Classification System }\end{array}$ & $\begin{array}{l}\text { All hospitals } \\
\mathbf{n}(\%)^{*}\end{array}$ & $\begin{array}{l}\text { Hospital 1 } \\
\mathbf{n}(\%)^{*}\end{array}$ & $\begin{array}{l}\text { Hospital 2 } \\
\mathbf{n}(\%)^{*}\end{array}$ & $\begin{array}{c}\text { Hospital 3 } \\
\mathbf{n}(\%)^{*}\end{array}$ \\
\hline 1 & $3564(18.9)$ & $776(18.1)$ & $745(18.3)$ & $2043(19.4)$ \\
2 & $1366(7.2)$ & $236(5.5)$ & $185(4.6)$ & $945(9.0)$ \\
3 & $7036(37.2)$ & $2035(47.5)$ & $1861(45.7)$ & $3140(29.7)$ \\
4 & $2077(11.0)$ & $342(8.0)$ & $378(9.3)$ & $1357(12.9)$ \\
5 & $2510(13.3)$ & $562(13.1)$ & $377(9.3)$ & $1571(14.9)$ \\
6 & $216(1.1)$ & $39(0.9)$ & $24(0.6)$ & $153(1.4)$ \\
7 & $355(1.9)$ & $78(1.8)$ & $82(2.0)$ & $195(1.8)$ \\
8 & $732(3.9)$ & $71(1.7)$ & $180(4.4)$ & $481(4.6)$ \\
9 & $8(0.0)$ & $4(0.1)$ & $2(0.0)$ & $2(0.0)$ \\
10 & $1044(5.5)$ & $140(3.3)$ & $235(5.8)$ & $669(6.3)$ \\
\hline Total & $\mathbf{1 8 9 0 8 ( 1 0 0 )}$ & $\mathbf{4 2 8 3 ( 1 0 0 )}$ & $\mathbf{4 0 6 9 ( 1 0 0 )}$ & $\mathbf{1 0 5 6 ( 1 0 0 )}$ \\
\hline
\end{tabular}

${ }^{*} \mathrm{n}=$ number of births in the group/total number of births in the hospital/s.

Table 2 presents differences in proportions of births among TGCS groups in the study hospitals. Groups 1 and 3 (nulliparous and multiparous women with single cephalic full-term pregnancy, with spontaneous labour without previous caesarean section) were the largest groups representing $56.1 \%$ of the total study population, ranging from $49.1 \%$ in hospital 3 to $65.6 \%$ in hospital 1 . The third largest group was group 5 (multiparous women with single cephalic full-term pregnancy, who had already undergone at least one caesarean section) which represented $13.3 \%$ of the study population, ranging from $9.3 \%$ in hospital 2 to $14.9 \%$ in hospital 3. Nulliparous (group 2) and multiparous women (group 4) with single cephalic full-term pregnancies, who required induction of labour or underwent prelabour caesarean section accounted for $7.2 \%$ and $11.0 \%$ of the total number of births, respectively. The largest variation between study hospitals was found in group 3 ranging from $29.7 \%$ in hospital 3 to $47.5 \%$ in hospital 1. Groups 6-10 accounted for $12.4 \%$ of all births.

A total of 4337 caesarean sections were performed, giving an overall caesarean section rate rate of $22.9 \%$, ranging from $20.6 \%$ in hospital 1 to $24.6 \%$ in hospital 3 (figure 1). Women in group 5 were the largest contributor to the overall caesarean section rates $(42.6 \%$, $1846 / 4337)$, ranging from $33.1 \%(283 / 855)$ in hospital 2 to $50.7 \%(448 / 884)$ in hospital 1 (figure 2, online supplementary table 2). The second and third strongest contributors were women with multiple pregnancies (group 8, $11.6 \%$ ) and those with cephalic preterm labour (group $10,8.1 \%)$. Among women in group 10 who gave birth by caesarean section, $54.4 \%(191 / 351)$ had a history of previous caesarean section. Groups 1 and 2 (singleton nulliparous women with cephalic full term pregnancies)

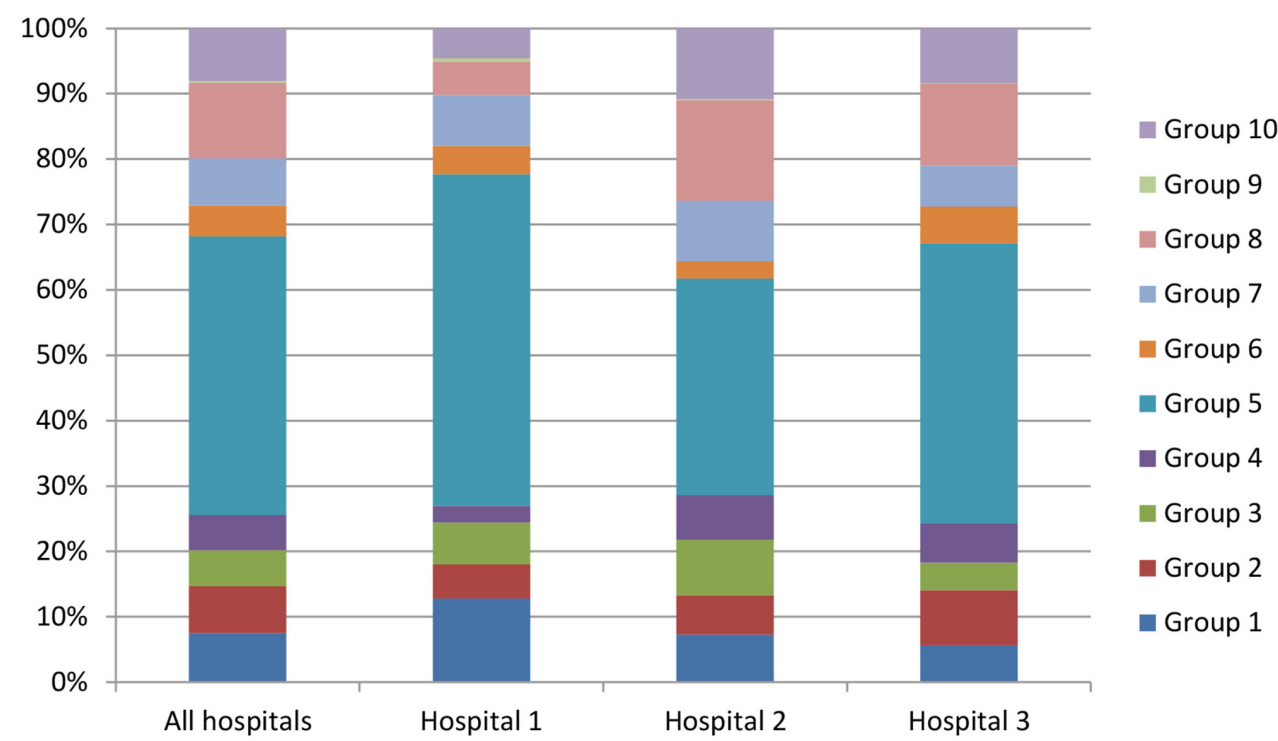

Figure 2 Contribution of each group in the Robson Ten Group Classification System to the overall caesarean section prevalence in the study hospitals $(n=4337)$. 
Table 3 Caesarean section rates in each group of the Robson Ten Group Classification System by the study hospitals $(\mathrm{n}=$ 18908)

\begin{tabular}{|c|c|c|c|c|c|}
\hline $\begin{array}{l}\text { Robson Ten Group } \\
\text { Classification System }\end{array}$ & $\begin{array}{l}\text { All hospitals } \\
\text { n (\%)* }\end{array}$ & $\begin{array}{l}\text { Hospital } 1 \\
\text { n }(\%)^{\star}\end{array}$ & $\begin{array}{l}\text { Hospital } 2 \\
\text { n }(\%)^{\star}\end{array}$ & $\begin{array}{l}\text { Hospital } 3 \\
\text { n }(\%)^{\star}\end{array}$ & $P$ valuest \\
\hline 1 & $324 / 3564$ (9.1) & $113 / 776$ (14.6) & 62/745 (8.3) & 149/2043 (7.3) & $<0.001$ \\
\hline 3 & 239/7036 (3.4) & $57 / 2035$ (2.8) & 73/1861 (3.9) & $109 / 3140(3.5)$ & 0.148 \\
\hline 4 & 236/2077 (11.4) & 23/342 (6.7) & $58 / 378$ (15.3) & 155/1357 (11.4) & 0.001 \\
\hline 7 & $312 / 355$ (87.9) & 69/78 (88.5) & 79/82 (96.3) & $164 / 195$ (84.1) & 0.017 \\
\hline 8 & $501 / 732(68.4)$ & 45/71 (63.4) & 132/180 (73.3) & $324 / 481(67.4)$ & 0.213 \\
\hline 9 & $8 / 8(100)$ & $4 / 4(100)$ & $2 / 2(100)$ & $2 / 2(100)$ & NAł \\
\hline 10 & $351 / 1044$ (33.6) & $41 / 140(29.3)$ & 92/235 (39.1) & 218/669 (32.6) & 0.095 \\
\hline
\end{tabular}

${ }^{*} \mathrm{n}=$ number of caesarean sections in the group/total number of births within the group.

$\dagger \mathrm{P}$ value from Pearson $\chi^{2}$ test comparing caesarean section rates by hospital in each group.

$\ddagger$ Not applicable because the rate of caesarean section is a constant.

combined contributed $14.7 \%$ to the overall caesarean section rates which was especially low in hospitals 2 and 3 with $13.3 \%$ and $14.1 \%$, respectively, and it was $18.0 \%$ in hospital 1.

Table 3 presents the caesarean section rates within each TGCS group in the study hospitals. Statistically significant differences in caesarean section rates between the TGCS groups were observed. The caesarean section rate was lowest $(3.4 \%)$ in the largest group (group 3). In the second and third largest groups, the caesarean section rates were $9.1 \%$ (group 1) and $73.5 \%$ (group 5), respectively. In groups 6,7 and 9 (breech presentation and abnormal fetal lies) more than $85 \%$ of births were by caesarean section. Significant differences in caesarean section rates between study hospitals were found among women in groups 1, 4, 5 and 7 (table 3 ).

Within group 5, 53.0\% (1330/2510) of women gave birth by prelabour caesarean section. Significant differences between hospitals were observed among women undergoing trial of vaginal birth after caesarean section (VBAC). VBAC ranged from 35.5\% (114/321) in hospital 1 to $65.3 \%(456 / 698)$ in hospital 3 (data not shown).

\section{DISCUSSION}

In Gaza, multiparous women with single full-term pregnancy, with at least one previous caesarean section (TGCS; group 5), women with multiple pregnancies (group 8) and women with preterm singletons in cephalic presentation (group 10) were the largest contributors to the overall caesarean section rate.

The study showed that group 5 was one of the three major contributors which is in line with findings in hospitals from the USA, Canada, France, Lithuania, Ethiopia, Tanzania and South Africa ${ }^{12-18}$ but the contributions of groups 8 and 10 in our study differ from previous studies in low-income and middle-income countries as well as in high-income countries. ${ }^{812-17}$ In most high-income countries, the major contributors to overall caesarean section rates were groups 5, 2 and $1 .{ }^{815}$ While in studies from low-income settings such as in Ethiopia, with extremely low caesarean section rates, the greatest contributors were groups 1,3 and $5 .^{14}$

Although the overall caesarean section rate in Gaza of $22.9 \%$ was relatively low compared with other continents such as $40.5 \%$ in Latin America, $32.3 \%$ in Northern America and $25.0 \%$ in Europe, it is still above the WHO criteria. ${ }^{14}$ According to Robson, differences between hospitals in the distribution of groups within the TGCS may be explained by differences in data quality, or be due to significant differences in important epidemiological variables or differences in clinical practice. ${ }^{12} 19$ In this cohort, the study population had similar sociodemographic and obstetrical characteristics. ${ }^{3}{ }^{11}$ The data collection was similar in all study hospitals, specific for this research purpose and comprised all births during the study period, reducing selection bias. ${ }^{311}$

The main contributor to the overall caesarean section rate was group 5 (women with singleton cephalic full-term pregnancy, who have undergone at least one caesarean section) having a caesarean section rate of $73.5 \%$, although group 5 only comprised $13.3 \%$ of all the women giving birth. In this study, the caesarean section rate in group 5 was comparable with those seen in Latin America and Lithuania, ${ }^{12}{ }^{13}$ but lower than those in the UK and Canada, and higher than those in Ireland, Norway and Sweden. ${ }^{20}$ Hospitals 1 and 3 had higher numbers of women in group 5, affecting the overall caesarean section rates. The large contribution of group 5 towards the total caesarean section rates in the study hospitals could be 
explained by some women having repeated caesarean section ( $>3$ times). In Gaza, there is no upper limit for the number of caesarean section per woman. Moreover, there were significant differences between the study hospitals in clinical trends for VBAC, where hospital 3 had the highest successful rate of VBAC $(65.3 \%)$ among women with previous one caesarean section. This was higher than in some studies, ${ }^{721}$ but in line with studies from Oman and Canada, reporting successful VBAC in $67.0 \%$ and $64.3 \%$, respectively, ${ }^{22} 23$ and in concordance with international standards or recommendations. ${ }^{24}$ However, the large number of primary caesarean sections in other TGCS groups will inevitably increase the number of women in group 5 which will thereby become an even more important contributor to the future overall caesarean section rate. Therefore, efforts to curb the trend of rising caesarean section rates need to address this group in order to be successful. Furthermore, significant differences between hospitals in VBAC rates suggest different obstetrical care practices in the study hospitals and demonstrate the ability to increase VBAC rates by appropriate management.

In contrast to previous studies, ${ }^{21} 25$ which took place in populations with a high proportion of nulliparous women, this study was conducted in a population with a high proportion of multiparous women. Robson et al and WHO expected the contributions of groups 1, 2 and 5 to make up two-thirds of the overall caesarean section rates, ${ }^{19} 26$ whereas in this study their contribution was less. In hospitals 2 and 3, the contributions of groups 1 and 2, to the overall caesarean section rates were very low with $13.3 \%$ and $14.1 \%$, respectively, although these groups make up $26.1 \%$ of the total study population. These rates were lower than in Ireland, Ethiopia and France. ${ }^{14} 2728$ On the other hand, the contributions of groups 8 and 10 were higher with $26.2 \%$ and $20.9 \%$, respectively, although these groups make up only $9.4 \%$ of the total study population in this study. This may suggest that obstetrical teams are good at dealing with uncomplicated pregnancies (group 1), while demonstrating less proficiency in dealing with complicated pregnancies, such as in groups 8 and 10. It appears that they prefer more invasive management, when faced with complicated obstetrics. This may be explained by having poor skills, poor equipment or by being understaffed to an extent that optimal care cannot be offered to these women. Also fear of litigation in the absence of professional medicolegal protection and a lack of routines to implement evidence-based clinical practice may contribute to explain their practice. Moreover, in this study, groups 1, 2 and 5 contributed to around $60 \%$ of the overall caesarean section rate which was similar to studies in Oman, Ireland and Iceland, ${ }^{20}{ }^{22}$ but less than other studies in Ethiopia, Italy and France. ${ }^{814} 15$

Women with multiple pregnancies (group 8) represented 3.9\% and those with preterm labour (group 10) $5.5 \%$ of the study population, with caesarean section rates of $68 \%$ and $34 \%$, respectively. These groups contributed more to the overall caesarean section rates than expected by Robson and those found in previous studies. ${ }^{12-18} 26$ This may be explained by the large number of women referred to the study hospitals (hospitals 1 and 3), as tertiary centres, due to in vitro fertilisation (IVF) treatment or other complications. ${ }^{26}{ }^{29}$ In Gaza, pregnancies resulting from IVF may be more likely to be delivered by caesarean section. Although the reason for this has not been studied, IVF pregnancies and babies may be considered more vulnerable and are therefore at higher risk of caesarean section. Furthermore, a history of previous caesarean section in $54.4 \%$ of women, who gave birth by caesarean section in group 10 did most probably increase the caesarean section rate in this group. The differences in caesarean section rates between the study hospitals were not statistically significant for groups 8 and 10. In previous studies, these two groups were small and contributed relatively little to the overall caesarean section rate. $^{1314202630}$

Focusing on the management of nulliparous women with single cephalic full-term pregnancies (groups 1 and 2) is important, as they represent one quarter of the obstetrical population in this study, and caesarean section in these groups will affect the future contribution of group 5 . In these groups, caesarean section is usually performed due to complications of labour such as dystocia or fetal distress and should be relatively low. ${ }^{13}$ Furthermore, variations in caesarean section rates between the study hospitals could be largely explained by variations in caesarean section rates among women in these two groups. ${ }^{20} \mathrm{In}$ this study, the average caesarean section rate in group $1(9.1 \%)$ was comparable with that reported in other studies, ${ }^{13} 202630$ but lower than in Ireland and France. ${ }^{15} 28$ However, significant differences between the study hospitals, as much as twofold (ranging from $7.3 \%$ in hospital 3 to $14.6 \%$ in hospital 1), showed differences in obstetrical practice in relation to the management of spontaneous labour.

Nearly half of the study population consisted of women from groups 3 and 4 (multipara single cephalic full-term with no previous caesarean section) which was higher than in previous studies. ${ }^{20} 212630$ These groups had less influence on caesarean section rates in all study hospitals as there were relatively few absolute medical indications for prelabour caesarean section and induction of labour was associated with low caesarean section rates. ${ }^{29}$

Therefore, reduction of primary caesarean sections is essential and has to be achieved by a multimodal approach including continuous staff training, increasing instrumental deliveries among low-risk groups and reducing the variations in delivered maternity care among Palestinian hospitals. One further aspect is to increase evidence-based practice among Palestinian obstetricians and midwives which might be one of the reasons for the unusually high rates of caesarean section in groups 8 and 10 . This study and ongoing continuous audits, including the examination of caesarean section indications within TGCS groups, would contribute to the continued surveillance of obstetrical practice in the government hospitals 
in Gaza. Furthermore, this study and ongoing local audits might have practical implications for health service planners to focus on the largest contributors to the overall caesarean section rate in order to standardise maternity care and improve quality of care.

\section{Strengths and limitations}

Strengths of this study include the prospective population-based cohort design. This study was the first to explore caesarean section rates in Palestine using the TGCS. All data were collected prospectively and therefore reducing the risk of information bias. Additionally, all women who gave birth in the three governmental hospitals during the study period were included, reducing the risk for selection bias.

The main limitation of the study was that women who gave birth in private hospitals or in governmental hospitals in the West Bank were not included. The study did not include caesarean section indications which may explain the differences among hospitals in some groups. ${ }^{14}$

\section{CONCLUSION}

Women in groups 5, 8 and 10 contributed the most to the overall caesarean section rate in the study hospitals. Significant variations in caesarean section rates between study hospitals were observed, and may reflect differences in obstetrical care. The efforts to reduce the overall caesarean section rate should be directed towards increasing VBAC in group 5 and reducing primary caesarean section.

\section{Author affiliations}

${ }^{1}$ Obstetrics Department, Al Shifa Hospital, Gaza, Palestine

${ }^{2}$ Faculty of Medicine, Institute for Clinical Medicine, University of Oslo, Oslo, Norway

${ }^{3}$ Intervention Centre, Oslo University Hospital Rikshospitalet, Oslo, Norway

${ }^{4}$ Department of Obstetrics, Oslo University Hospital, Ullevål, Oslo, Norway

${ }^{5}$ Department of Health Management and Health Economics, Institute of Health and

Society, University of Oslo, Oslo, Norway

${ }^{6}$ Faculty of Pharmacy, Nursing, and Health Professions, Birzeit University, Birzeit, State of Palestine

${ }^{7}$ Faculty of Medicine, Islamic University of Gaza, Gaza, State of Palestine

${ }^{8}$ Obstetrics Department, Palestine Medical complex, Ramallah, State of Palestine

${ }^{9}$ Obstetrics Department, Al Aqsa Hospital, Gaza, State of Palestine

${ }^{10}$ Oslo Centre for Biostatistics and Epidemiology, Research Support Services, 0slo University Hospital, Oslo, Norway

${ }^{11}$ Department of Gynecology, Oslo University Hospital, Oslo, Norway

Acknowledgements The authors would like to extend the best thanks and gratitude to the Norwegian Research Council for funding this study. They also thank the Palestinian Ministry of Health for supporting the study and appreciate the effort and time spent by research teams for data collection.

Contributors MWZ: in-charge of data collection, participated in staff training on data registration and entry, statistical analysis for the dataset, participated in interpretation of the results and drafted the manuscript. KL: study design, protocol and research tool development, participated in staff training on data registration and entry and commented on the manuscript. SH: study design, collaborated in the preparation of the protocol and research tool development, data collection, participated in staff training on data registration and entry and commented on the manuscript. EF: study design, protocol development and commented on the manuscript. ML: commented on the manuscript. KMZ and HYA-M: data collection, participated in staff training on data registration and entry and commented on the manuscript. BB: participated in interpretation of the results, revised the medical
English language and commented on the manuscript. RSF: statistical analysis for the dataset, participated in interpretation of the results and commented on the manuscript. ÅV: study design, protocol and research tool development and participated in staff training on data registration and entry, participated in interpretation of the results and commented on the manuscript. All authors revised, comments and approved the final version.

Funding This study was funded by the Norwegian Research Council through 0slo University Hospital (grant number: 234452/14).

Disclaimer The funding source had no role in the study design, data collection, data analysis, data interpretation or writing of the manuscript. All authors had full access to all the data in the study and can take responsibility for the integrity and accuracy of the data analysis and they had final responsibility for the decision to submit for publication

Competing interests None declared.

Patient consent Not required.

Ethics approval This study was approved by the Palestinian health research

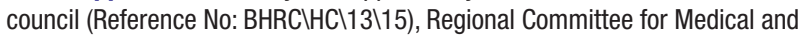
Health Research Ethics in South-Eastern Norway (REK 2014/1727) and the Norwegian Data Inspectorate (17/00082-2/GRA). Oslo University Hospital signed an agreement with the Palestinian Ministry of Health which approved conducting the study within their facilities. The project was done in accordance with common rules for healthcare services in Palestine and Norway regarding, eg, privacy.

Provenance and peer review Not commissioned; externally peer reviewed.

Data sharing statement No additional data are available.

Open access This is an open access article distributed in accordance with the Creative Commons Attribution Non Commercial (CC BY-NC 4.0) license, which permits others to distribute, remix, adapt, build upon this work non-commercially, and license their derivative works on different terms, provided the original work is properly cited, appropriate credit is given, any changes made indicated, and the use is non-commercial. See: http://creativecommons.org/licenses/by-nc/4.0/.

\section{REFERENCES}

1. Betrán AP, Ye J, Moller AB, et al. The increasing trend in caesarean section rates: global, regional and national estimates: 1990-2014. PLoS One 2016;11:e0148343.

2. Bragg F, Cromwell DA, Edozien LC, et al. Variation in rates of caesarean section among English NHS trusts after accounting for maternal and clinical risk: cross sectional study. BMJ 2010;341:c5065.

3. Zimmo M, Laine K, Hassan S, et al. Differences in rates and odds for emergency caesarean section in six Palestinian hospitals: a population-based birth cohort study. BMJ Open 2018;8:e019509.

4. Organization WH. Appropriate technology for birth. Lancet 1985;2:436-7.

5. Robson MS. Classification of caesarean sections. Fetal Matern Med Rev 2001;12:23-39.

6. Betran AP, Torloni MR, Zhang JJ, et al. WHO Statement on caesarean section rates. BJOG 2016;123:667-70.

7. Chong C, Su LL, Biswas A. Changing trends of cesarean section births by the Robson Ten Group Classification in a tertiary teaching hospital. Acta Obstet Gynecol Scand 2012;91:1422-7.

8. Ciriello E, Locatelli A, Incerti M, et al. Comparative analysis of cesarean delivery rates over a 10-year period in a single Institution using 10-class classification. J Matern Fetal Neonatal Med 2012;25:2717-20.

9. Ministry of Health. In: Ramallah P, Health status, palestine 2015163 : State of Palestine, 2016:50-3.

10. United Nations Population Fund, UNFPA. Palestine 2030 demographic change: opportunities for development, December 2016. 55: United Nations Population Fund, UNFPA:86.

11. Hassan $S$, Vikanes $A$, Laine $K$, et al. Building a research registry for studying birth complications and outcomes in six Palestinian governmental hospitals. BMC Pregnancy Childbirth 2017;17:112.

12. Robson M. The Ten Group Classification System (TGCS) - a common starting point for more detailed analysis. BJOG 2015;122:701-01.

13. Barčaitė E, Kemeklienė G, Railaite DR, et al. Cesarean section rates in lithuania using robson ten group classification system. Medicina 2015;51:280-5.

14. Tura AK, Pijpers O, de Man M, et al. Analysis of caesarean sections using Robson 10-group classification system in a university 
hospital in eastern Ethiopia: a cross-sectional study. BMJ Open 2018;8:e020520.

15. Lafitte AS, Dolley P, Le Coutour X, et al. Rate of caesarean sections according to the Robson classification: Analysis in a French perinatal network - Interest and limitations of the French medico-administrative data (PMSI). $J$ Gynecol Obstet Hum Reprod 2018;47:39-44.

16. Roberge S, Dubé E, Blouin S, et al. Reporting caesarean delivery in quebec using the robson classification system. J Obstet Gynaecol Can 2017;39:152-6.

17. Kacerauskiene J, Bartuseviciene E, Railaite DR, et al. Implementation of the Robson classification in clinical practice:lithuania's experience. BMC Pregnancy Childbirth 2017;17:432.

18. Hehir MP, Ananth CV, Siddiq Z, et al. Cesarean delivery in the United States 2005 through 2014: a population-based analysis using the Robson 10-Group Classification System. Am J Obstet Gynecol. In Press. 2018;219:105.e1-105.e11.

19. World Health Organization. Robson classification: implementation manual. Geneva: World Health Organization, 2017.

20. Brennan DJ, Robson MS, Murphy M, et al. Comparative analysis of international cesarean delivery rates using 10-group classification identifies significant variation in spontaneous labor. Am J Obstet Gynecol 2009;201:308.e1-308.e8.

21. Nakamura-Pereira M, do Carmo Leal M, Esteves-Pereira AP, et al. Use of Robson classification to assess cesarean section rate in Brazil: the role of source of payment for childbirth. Reprod Health 2016;13:128.
22. Kazmi T, Saiseema S, Khan S. Analysis of Cesarean Section Rate - According to Robson's 10-group Classification. Oman Med J 2012;27:415-7.

23. Grobman WA, Lai Y, Landon MB, et al. The change in the VBAC rate: an epidemiologic analysis. Paediatr Perinat Epidemiol 2011;25:37.

24. Russillo B, Sewitch MJ, Cardinal L, et al. Comparing rates of trial of labour attempts, VBAC success, and fetal and maternal complications among family physicians and obstetricians. J Obstet Gynaecol Can 2008;30:123-8.

25. Maneschi F, Algieri M, Perrone S, et al. Cesarean 10-group classification: a tool for clinical management of the delivery ward. Minerva Ginecol 2015;67:389-95.

26. Perinatal Services BC. Examining cesarean delivery rates in British Columbia using the Robson ten classification. Part 1: understanding the ten groups. 1. Vancouver, BC, 2011.

27. Le Ray C, Prunet C, Deneux-Tharaux C, et al. [Robson classification: a tool for assessment of caesarean practices in France]. $J$ Gynecol Obstet Biol Reprod 2015;44:605-13.

28. Sinnott SJ, Brick A, Layte R, et al. National variation in caesarean section rates: a cross sectional study in Ireland. PLoS One 2016;11:e0156172.

29. Robson M, Hartigan L, Murphy M. Methods of achieving and maintaining an appropriate caesarean section rate. Best Pract Res Clin Obstet Gynaecol 2013;27:297-308.

30. Betrán AP, Gulmezoglu AM, Robson M, et al. WHO global survey on maternal and perinatal health in Latin America: classifying caesarean sections. Reprod Health 2009;6:18. 